\section{New area in malaria}

\section{By Lev Osherovich, Senior Writer}

Development of malaria therapeutics has been stymied by poor understanding of drug targets and limited corporate interest in picking up preclinical compounds with therapeutic promise. At the same time, the parasite has shown mounting resistance to older drugs, making drugs against new targets ever more urgent.

Now, a trio of articles describes a range of drug discovery approaches that may yield new types of antimalarial agents. ${ }^{1-3}$ The proof-of-principle compounds identified in the studies are not yet ready to enter the clinic, but they could serve as starting points for designing improved compounds using SAR analysis, according to the authors.

With clinical testing infrastructure already in place from previous trials and financial incentives available from not-for-profits such as Medicines for Malaria Venture (MMV), the challenge for industry is to advance the new compounds through the bottleneck of corporate preclinical development into not-for-profit-sponsored clinical testing programs.

The studies take contrasting approaches to finding new compounds and target distinct steps in the complex life cycle of Plasmodium falciparum, the protozoan parasite that causes about $80 \%$ of malaria cases. (The other $20 \%$ of cases are caused by other Plasmodium parasites.)

P. falciparum's life cycle has several windows of vulnerability to therapeutics.

After initial human infection, parasites spend much of their time inside red blood cells, feeding on hemoglobin. The bugs replicate in the immunological safe haven of the red blood cell, periodically bursting forth to infect new blood cells. Chloroquine, the most widely used malaria drug, blocks this asexual phase of the organism's life cycle.

However, chloroquine-resistant strains have emerged, making it necessary to use combinations of more costly drugs such as artemisinin derivatives. Developing a cheap, first-line replacement for chloroquine that does not lead to resistance is thus a major aim of philanthropically funded malaria research. ${ }^{4}$

Two independent teams have taken a stab at this goal, using a cell-based screening approach to identify new compounds with equal or better efficacy than chloroquine and artemisinin.

A third study looks at new targets involved in another critical step in the malaria cycle, gametogenesis. This study shows that targeting gamete maturation with compounds that affect cyclic guanosine monophosphate (cGMP) signaling could be an opportunity to block Plasmodium transmission.

\section{A wide net}

The first study, published in the Proceedings of the National Academy of Sciences, was conducted by an industry-academic partnership led by Elizabeth Winzeler, associate professor of cell biology at The Scripps Research Institute. Winzeler is also a department head at the Genomics Institute of the Novartis Research Foundation, a not-for-profit research institute funded by Novartis AG.

Her team screened Novartis' library of about 1.7 million compounds for inhibitors of Plasmodium DNA replication in cultured red blood cells, using a fluorescence-based high throughput readout. The screen yielded about 6,000 compounds with an $\mathrm{IC}_{50}$ of $1.25 \mu \mathrm{M}$ or better.

Although this concentration translates to about 1,000-fold lower potency than current drugs, the researchers reasoned that even weak inhibitors could be a foot in the door to new chemical scaffolds. Moreover, 648 compounds had $\mathrm{IC}_{50}$ values of less than $100 \mathrm{nM}$, approaching the range of existing drugs.

The hits included most marketed antimalarial drugs, suggesting that the screening method is likely not to miss equivalently potent new compounds.

Winzeler's group then used computational methods based on structural similarities, data from previous screens of the Novartis compound library and the Medical Subject Headings (MeSH) system of functional classification to group the compounds into categories. The hits fell into about 530 structural categories with at least 31 known types of biological effects.

The categories included familiar antimalarial drug types, as well as protein synthesis inhibitors, antineoplastic agents, antihypertensive agents and antifolate drugs. The majority of compounds, however, were both structurally and functionally new.

Winzeler told SciBX that because only a few specific targets in Plasmodium have been adequately characterized, casting a wide net for relatively low affinity inhibitors in a cell-based growth assay is the best starting point for drug discovery.

"This is a method to get a large number of antimalarial hits," she said. "We've found things that have not been characterized as antimalarial drugs."

Winzeler noted that among the weak hits were compounds with "relatively potent activity" that will be further characterized in a rodent model of Plasmodium infection. The most promising compounds have not yet been published, she said.

The work was partially funded by not-for-profits including MMV and The Wellcome Trust. Most of the compounds identified in the screen belong to Novartis and are neither patented nor available for licensing, according to Winzeler. Some of the compounds identified in the study are patented by other companies, as the library contains a large collection of compounds synthesized without regard to ownership, she said.

\section{Keep it natural}

Meanwhile, a more narrowly focused screen of bacterial extracts identified a compound with potent antimalarial activity. The study 
was published in Public Library of Science ONE by a team led by Karine Le Roch, assistant professor of cell biology and neuroscience at the University of California, Riverside. The compound-salinosporamide A-is licensed to Nereus Pharmaceuticals Inc. and is in Phase I testing for solid tumors and multiple myeloma (MM).

Le Roch told SciBX that the best antimalarial drugs, such as artemisinin and the chloroquine predecessor quinine, are derived from natural compounds or secondary metabolites made by plants and microbes. One advantage of natural products is the lower likelihood of Plasmodium developing resistance, Le Roch said. Thanks to natural selection, she noted, "microorganisms have been doing the work of fighting resistance throughout the course of evolution."

Le Roch's team used the same red blood cell-based assay as Winzeler's group to screen extracts from 80 marine actinomycete bacteria for antimalarial activity. Actinomycetes have a long track record of making medically useful natural compounds, such as the antibiotic actinomycin.

The most potent antimalarial extract identified in the paper was from Salinospora tropica, which previously had been shown to make salinosporamide A, a potent proteasome inhibitor. The researchers repeated their study with purified salinosporamide A and obtained an $\mathrm{IC}_{50}$ of $11.4 \mathrm{nM}$, comparable to artemisinin.

Le Roch told SciBX that salinosporamide A inhibits the proteasome and arrests the organism during DNA replication, perhaps blocking the degradation of cell-cycle checkpoint proteins. She noted that similar antimalarial activity has been reported with Velcade bortezomib. ${ }^{5}$

Velcade is marketed by Millennium Pharmaceuticals Inc., a subsidiary of Takeda Pharmaceutical Co. Inc., and Johnson \& Johnson to treat mantle cell lymphoma and MM.

Le Roch is collaborating with Nereus to further characterize the compound in a rodent model of malaria and to optimize the selectivity of salinosporamide A for Plasmodium proteasomes rather than those of the human host.

Nereus CEO Kobi Sethna told SciBX the company is primarily focused on cancer and that the antimalarial activity of its compound is "a very new development for us."

Le Roch and Nereus have applied for a collaborative MMV grant to further develop the compound for malaria.

\section{Premature exflagellation}

A third paper describes an even more focused approach-targeting specific enzymes involved in gametogenesis, the sexual phase of Plasmodium's life cycle that is required for transmission of the parasite from humans to mosquitoes.

After being taken up during the mosquito's blood meal, male and female Plasmodium cells mate to produce the infectious sporozoites that are passed along to the next human host. Mating, which takes place inside of mosquitoes, involves the emergence of Plasmodium gametes from human red blood cells sucked up by the mosquito, followed by a morphological change called exflagellation.
The study was published in Public Library of Science Biology by a team led by David Baker, reader in parasite molecular biology at the London School of Hygiene \& Tropical Medicine.

Building on previous studies, the team tested whether compounds that affect cGMP signaling could influence sexual maturation of $P$. falciparum in a cell-culture assay.

The first target attacked by Baker's team was the cGMP phosphodiesterase (cGMP-PDE), which degrades cGMP. Indeed, blocking cGMP-PDE accelerated Plasmodium gametogenesis.

Baker's team found that a preclinical cGMP-PDE inhibitor called zaprinast led to premature exflagellation. Zaprinast functions similarly to approved drugs such as Viagra sildenafil, a PDE inhibitor that is marketed by Pfizer Inc. to treat erectile dysfunction and pulmonary arterial hypertension (PAH).

In contrast, the team found that inhibiting another enzyme, cGMP-dependent protein kinase-1 (PKG-1), blocked gametogenesis. The inhibitor, a trisubstituted pyrrole (4-[2-(fluorophenyl)-5(1-methylpiperidine-4-yl)-1H pyrrol-3-yl] pyridine), was obtained from Merck \& Co. Inc. and had previously been in development to treat Eimeria, a poultry parasite, according to Baker. Merck did not disclose the development status of the compound.

Together, the findings suggest that gametogenesis involves high cGMP levels and the action of PKG-1. However, the target of PKG-1 is not yet known.

Even though the approved malaria drugs
-David Baker, Tropical Hygiene \& Tropical Medicine work by blocking the asexual phase of Plasmodium replication, Baker told SciBX that his study opens up new stages of the parasite's life cycle to therapeutic intervention.

"If we can inhibit PKG-1, the first step of gametogenesis cannot take place," he said. "The implication is that we might be able to block transmission of the parasite."

Baker said unpublished data from his lab suggest that PKG-1 is also needed for asexual replication, making the enzyme a more attractive target for drug development. This is because a PKG-1 inhibitor could block both phases of the Plasmodium life cycle, potentially preventing both disease and transmission.

However, Baker thinks the PKG-1 inhibitor used in his study is not yet suitable as a drug because of potential toxicity. His next step is to screen large compound libraries, such as the one used by Winzeler's group, for more selective PKG-1 inhibitors that target the Plasmodium enzyme without blocking the human version of the enzyme.

The library that Winzeler's group used is not publicly accessible.

While the PKG-1 approach is fleshed out, cGMP-PDE may be a better target for now. In a previous study, Baker's team reported that deletion of Plasmodium's c GMP-PDE gene led to premature exflagellation and thus prevented emergence from red blood cells. ${ }^{6}$ Thus, a therapeutic that makes Plasmodium prematurely exflagellate before reaching the mosquito could prevent carriers from passing on the parasite.

"The gametocytes must absolutely remain quiescent until they arrive in the mosquito," said Baker. "If they emerged in the human host they would die." 
He suspects that PDE inhibitors such as Viagra could be repurposed to disrupt malaria transmission.

\section{Global perspectives}

Melinda Moree, an independent global health consultant who was formerly director of the PATH Malaria Vaccine Initiative, told SciBX that the Baker study could be complementary to efforts to develop transmission-blocking malaria vaccines. However, she cautioned that social and economic factors make it difficult to test prophylactic compounds.

"It's going to be exceedingly difficult to get money for something that doesn't treat the disease," Moree said. "However, with global sights set on eradication, we'll have to target transmission too."

Moree told SciBX that the best place for PKG-1 inhibitors may be "as part of a combination, along with a therapeutic" that treats the asexual phase of Plasmodium's life cycle. Such therapeutics could include compounds derived from Winzeler's study.

Timothy Wells, CSO of MMV, said Baker's study could indeed open up new therapeutic real estate in malaria.

"The fact that he's got an assay and enzyme that plays an important role in gametogenesis is exciting," said Wells. "The number of targets specifically linked to gametogenesis is really quite low."

He suggested that Baker and Winzeler could team up to put Novartis' drug library through Baker's gametogenesis assay, as well as to use more traditional SAR methods to synthesize next-generation inhibitors.

Winzeler's group recently used library screening to identify an inhibitor of a different Plasmodium kinase, calcium-dependent protein kinase 1 , which is involved in asexual replication. ${ }^{7}$

"The next step is to pair Baker up with people who really understand the kinase space," said Wells.

He noted that the bottleneck in malaria drug development is access to the medicinal chemistry resources that pharma musters for more lucrative drug development. The Winzeler and Baker studies make this space more attractive for serious efforts by pharma, Wells told SciBX.
"A lot of biology has been done to validate targets, but in academia there's only a small number of workers" with the needed medicinal chemistry experience to shepherd compounds from proof of concept to clinical candidate.

"These papers give us justification to say 'Give us more chemists, please,' said Wells.

\section{REFERENCES}

1. McRobert, L. et al. PLoS Biol.; published online June 3, 2008; doi:10.1371/journal.pbio.0060139

Contact: David A. Baker, London School of Hygiene \& Tropical Medicine, London, U.K.

e-mail: david.baker@Ishtm.ac.uk

2. Prudhomme, J. et al. PLoS ONE; published online June 4, 2008; doi:10.1371/journal.pone.0002335

Contact: Karine Le Roch, University of California, Riverside, Calif. e-mail: karine.leroch@ucr.edu

3. Plouffe, D. et al. Proc. Natl. Acad. Sci. USA; published online June 9, 2008; doi:10.1073/pnas.0802982105

Contact: Elizabeth A. Winzeler, The Scripps Research Institute, La Jolla, Calif.

e-mail: winzeler@scripps.edu

4. Medicines for Malaria Venture. Product profiles for antimalarial drugs. <http://www.mmv.org/IMG/pdf/PRODUCT_PROFILE_with_logo.pdf> (July 2006)

5. Reynolds, J.M. et al. BMC Clin. Pharmacol. 7, 13 (2007)

6. Taylor, C.J. et al. Mol. Microbiol.69, 110-118 (2008)

7. Kato, N. et al. Nat. Chem. Biol. 4, 347-356 (2008)

COMPANIES AND INSTITUTIONS MENTIONED Genomics Institute of the Novartis Research Foundation, La Jolla, Calif.

Johnson \& Johnson (NYSE:JNJ), New Brunswick, N.J. London School of Hygiene \& Tropical Medicine, London, U.K. Medicines for Malaria Venture, Geneva, Switzerland Merck \& Co. Inc. (NYSE:MRK), Whitehouse Station, N.J. Millennium Pharmaceuticals Inc. (NASDAQ:MLNM), Cambridge, Mass.

Nereus Pharmaceuticals Inc., San Diego, Calif.

Novartis AG (NYSE:NVS; SWX:NOVN), Basel, Switzerland

PATH Malaria Vaccine Initiative, Bethesda, Md.

Pfizer Inc. (NYSE:PFE), New York, N.Y.

The Scripps Research Institute, La Jolla, Calif.

Takeda Pharmaceutical Co. Ltd. (Tokyo:4502), Osaka, Japan

University of California, Riverside, Calif.

The Wellcome Trust, London, U.K. 